\title{
LOOKS LIKE BUT DOES IT FEEL LIKE? INVESTIGATING THE INFLUENCE OF MASS PROPERTIES ON USER PERCEPTIONS OF RAPID PROTOTYPES
}

\author{
H. Felton ${ }^{\otimes}$, J. Yon and B. Hicks \\ University of Bristol, United Kingdom \\ $\triangle$ harry.felton@bristol.ac.uk
}

\begin{abstract}
Prototyping is a key part of the design process, with artefacts increasingly fabricated using 3D printing methods. However, these printed parts often lack internal structure and the mass properties of the artefact - mass, balance and moments of inertia - differ from the design. It is hypothesised that a stakeholder's assessment of a design is affected by this misrepresentation. The work presented demonstrates that mass properties have a significant effect on stakeholder perception of prototypes. This is done through a study of University of Bristol students and consultation with industry.
\end{abstract}

Keywords: prototyping, 3D printing, rapid prototyping, design tools, user experience

\section{Introduction}

Prototypes are used throughout the design community to infer and present design ideas and concepts, for the purposes of communication and learning (Yang, 2004). It is often an activity carried out throughout a part or assembly's design cycle. Final stage prototypes typically reflect the intended design in fine detail, with aesthetics and function represented to a near production level. In the formative stages of prototyping it may not be feasible or necessary to produce such an implementation prototype due to time, cost or insufficient design detail (Jensen et al., 2016). Correspondingly these prototypes may lack potentially important qualities of a design. For example, if a prototype is 3D printed, as many commonly are, it is likely that the representation of mass properties - mass, balance and inertia - will be poor. There are several reasons for this related to the materials used, the internal structure of the artefact and the CADCAM tools.

This paper seeks to investigate whether poor replication of mass properties is an issue that influences user perceptions of rapid prototypes (RPs) and hence user feedback in design processes. A review of current prototyping literature, a study of students at the University of Bristol and a consultation of industry are presented. The paper findings are then summarised, and work suggested that would enable the replication of mass properties in $3 \mathrm{D}$ printed artefacts.

\section{Product prototyping}

Prototyping encompasses the activity of producing a prototype artefact and learning from interaction with the prototype (Buchenau and Suri, 2000). This can be completed in the physical or virtual 
domain, and design cycles commonly incorporate some form of physical and virtual prototyping already (Gibson et al., 2004). The focus of the report is physical prototypes - which can be loosely defined as a tangible artefact that is intended to convey information on a design. Presented within this section is a discussion around the purpose of prototyping, the types of prototype produced, and how physical prototype artefacts are made.

\subsection{Purpose of prototyping}

Prototyping can be a design tool that can be used to achieve many potential aims and objectives. Fundamentally, prototypes are intended to accelerate the design cycle and streamline learning for the design engineer and/or product stakeholder. This is done by enhancing the ability of those parties to evaluate and understand a design. They allow the simulation of a design to answer specific questions concretely (Yang, 2004), or raise new questions that were not otherwise obvious. K. Schneider summarises this by stating

"A prototype is just another representation for requirements and solution knowledge" (Schneider, 1995).

However, the knowledge that is hoped to be conveyed and understood through prototyping activities can differ across a design cycle and across industries. Camburn et al. raised four key purpose designations outlined as:

- Refinement - A prototype intended to gradually improve a design;

- Communication - A prototype intended to share knowledge regarding the design and use to stakeholders;

- Exploration - A prototype intended to encourage creativity in finding new design concepts; and,

- Active Learning - A prototype intended to allow the stakeholders to advance their understanding of the design space (Camburn et al., 2017).

Through inspection of these four purposes it can be observed that prototyping activities can be used throughout the design cycle. An important clarification is that the purposes stated are not distinct; they are merely reasons why a designer may choose to prototype. It should also be noted that several of the four purposes can often be met in a single prototype.

\subsection{Types of prototype}

Once the anticipated purpose of a prototype is defined, a designer must decide upon the form the prototype should take. Although the methods by which the prototype will be fabricated are considered in more depth in Section 2.3, defining the type of prototype that needs to be produced is an important step. From the analysis of why designers prototype, prototypes can generally be considered to fall into three groups, given in Table 1 .

Table 1. Prototype definitions from (Houde and Hill, 1997)

\begin{tabular}{|c|c|}
\hline Prototype & Definition \\
\hline Look and Feel & A prototype that answers questions regarding the sensory experience of using an artefact. \\
\hline Role & $\begin{array}{r}\text { A prototype that answers questions regarding the purpose and/or function that an } \\
\text { artefact serves in a user's life. }\end{array}$ \\
\hline Implementation & $\begin{array}{c}\text { A prototype that functions as the final artefact is intended to, either fully or through } \\
\text { demonstration of a single technique. }\end{array}$ \\
\hline
\end{tabular}

These three definitions represent a triangular space in which all prototypes should lie. This is demonstrated graphically in the Figure 1. Central to this space is a zone of integrated prototypes - a set of prototypes that involve each of the three sub-types to form a prototype more like that of the intended product than otherwise possible. Generally, the use of integrated prototypes will come later in the design cycle due to their increased complexity and cost. 


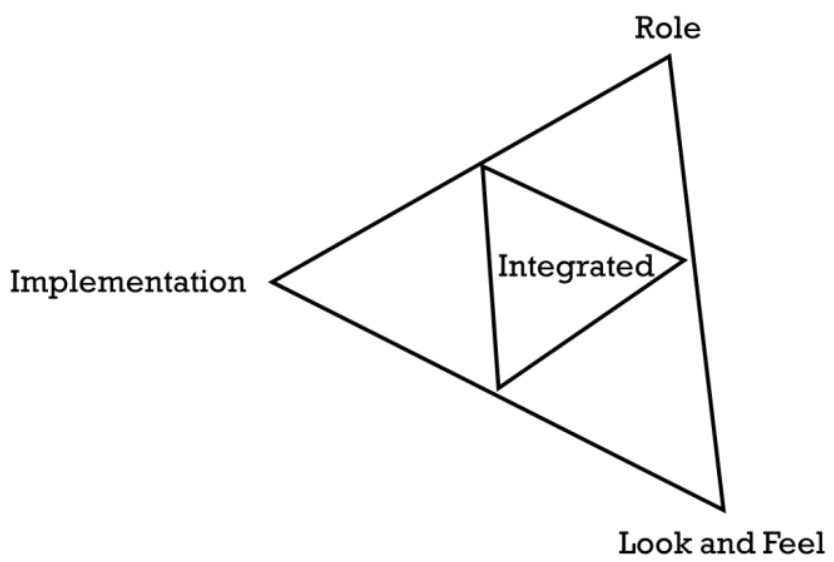

Figure 1. Prototype space as defined by S. Houde and C. Hill (Houde and Hill, 1997)

The focus of this paper will be those prototypes that are intended to be look and feel prototypes, to enhance the "feels-like" aspects of the artefact. It's also thought that the method will be best applied to purposes of refinement, communication and active learning (as previously defined).

\subsection{Methods of prototype fabrication}

It is important to recognise that prototypes can be both virtual and physical in form. This is true even for physical products, where a virtual prototype may be used to simulate function, represent geometry or serve some other purpose. With the advent of augmented and virtual reality, as outlined by Coutts, Wodehouse and Robertson (Coutts et al., 2019), these categorisations are being blurred and it is thought this space will continue to develop further. However, for the purpose of the work undertaken here within, only physical prototypes will be considered and discussed. A brief summary of several of the most common physical prototyping methods are presented within this section, discussed and compared in more detail by Mathias et al. (Mathias et al., 2018).

\subsubsection{Cardboard based prototypes}

Cardboard based prototypes are common practice in industry (see Figure 2). They offer a means of prototyping that is free-form, requires little expertise, and is low-fidelity. Their main purpose is to provide a looks-like prototype, often lacking in functionality. Dyson commonly use cardboard in the early stages of design and prototyping (James Dyson Foundation, 2019). This is due to the rapid fabrication times, low cost and accessibility of the technology. Fine details can be generated, and the aesthetics can be improved using paint or decals.

\subsubsection{Construction kits}

Construction kits are commonplace in industry - and are often used as toys. Examples such as LEGO, Duplo and K'Nex are well known and can be used to create complex shapes and structures, all be it with a discrete building capability. Initially considered toys, these kits have been developing momentum as design tools for engineers and hobbyists alike (Mathias et al., 2019). LEGO Technic kits now allow users to create mechanisms and dynamic models that can be interacted with.

\subsubsection{Classical manufacturing techniques}

It is, of course, possible to produce a prototype using the manufacturing methods anticipated to be used for the final product design. For example, a wheel hub that is intended to be made using 5-axis CNC (Computer Numerical Control) machine, can be prototyped using a 5-axis CNC machine using the same material and machine code as production. This would allow, for example, structural testing of the part for a direct comparison to required performance. In some industries this is a requirement. However, this is often an expensive process requiring significant expertise. In instances where tooling is used, post-fabrication design changes are particularly expensive. (Lennings, 1997) 


\subsubsection{D printing (additive manufacturing)}

3D printing, otherwise known as Additive Manufacturing (AM) and RP, is a means of producing a prototype in a highly automated way. Several methods exist in order to do this, though Material Extrusion (MEX) methods, also known as Fused Deposition Modelling (FDM) and Fused Filament Fabrication (FFF) are still the most common (Simonot et al., 2019). MEX has been used for many years but has recently become a much more accessible technology due to a patent held by Stratasys expiring. This has led to several companies creating machines that take advantage of the technology. Figure 2 shows the range of applications of MEX prototypes in industry.

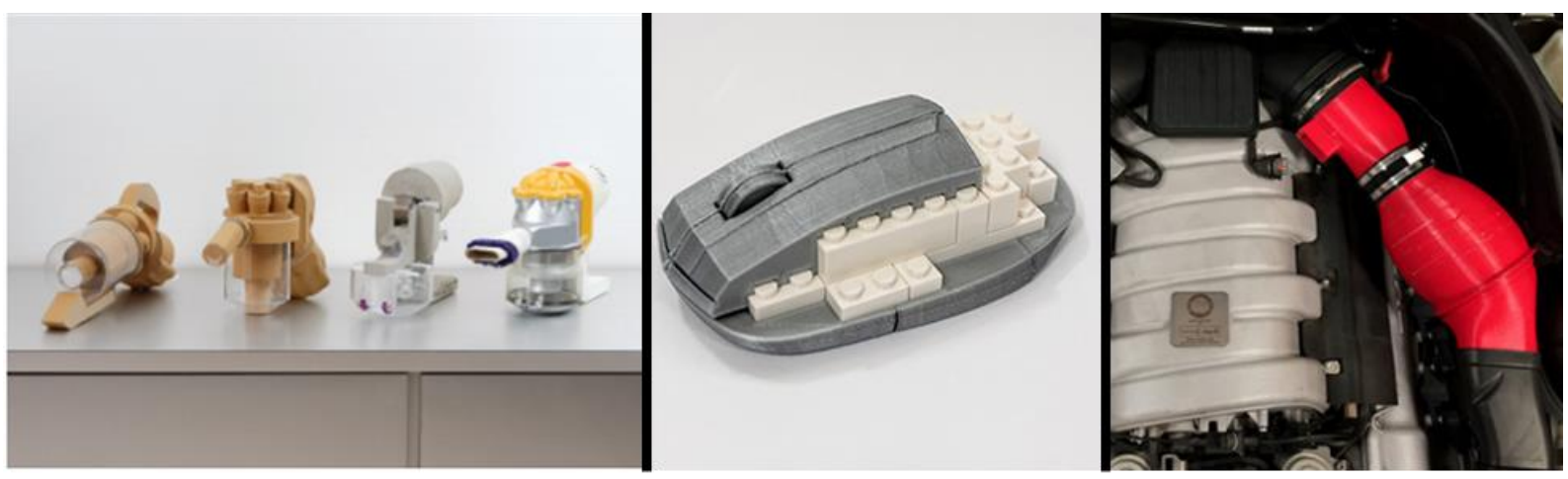

Figure 2. Examples of different prototyping methods; cardboard (James Dyson Foundation, 2019), construction kit (Mathias, 2018) and MEX (Ultimaker, 2019)

MEX printing allows the fabrication of moving assemblies, structural parts and flexible joints all in a single print. Material capability ranges from low-cost polymers, such as PLA and ABS, through to metals and ceramics - see Desktop Metal (Desktop Metal, 2019) and Markforged Metal X (Markforged, 2019) for more information. The technology is used by both hobbyists and professionals and is continuing to grow in use (Moreau, 2018). It offers significant freedoms of manufacture though, as the volume of material extruded is directly proportional to time and cost of manufacture, fabricated parts often contain a low-density internal structure.

\section{The perception of mass}

As previously stated, a significant amount of prototyping activity is completed using MEX produced artefacts. The ability for the process to be highly automated with short-turnaround times allows parts to be manufactured with ease, reducing the risk of design fixation due to fabrication time as outlined by V. K. Viswanathan and J. S. Linsey (Viswanathan and Linsey, 2013). However, these parts often lack representative mass properties of the intended design. This is due to MEX parts regularly having a low-density internal structure, as previously stated. These structures are used due to the process cost and time being directly proportional to volume of material printed.

For certain prototyping activities the effect of misrepresenting mass properties would be negligible. For example, if a prototype is to sit on a counter to be visually observed, it does not need to have representative mass properties. This can also be true of some static parts and assemblies. However, it is thought anything intended for dynamic applications or that will be interacted with by users requires representative mass properties to be mapped to a prototype at some point in the design cycle. Currently, it is thought that this more commonly occurs in the later stages of design, where changes are costly, and a designer(s) is/are likely to have fixated on a design.

It is hypothesised that the mass properties of a part - the mass, balance and inertia of a part - can influence a user's perception of a design earlier in the design cycle. For example, when exploring the design space and communicating design concepts. It is suggested any method should be automated as far as reasonably practicable - to reduce the bias caused by fabrication time, as previously discussed above and noted by Viswanathan and Linsey (Viswanathan and Linsey, 2013).

In literature, the only reference to the value of prototyping mass that has been found by the authors was in "What do prototypes prototype?" (Houde and Hill, 1997). In this paper Houde and Hill state 
that a brick can be a prototype if it communicates a notion of the mass of the intended design. This was as far as the paper took this concept. Otherwise, the importance of mass properties in prototyping has been found to be a neglected topic in academia, with references implied rather than explicit.

The work detailed herein aims to investigate the influence of mass properties on user perceptions of RPs explicitly through a user study and consultation with industry.

\section{Study design}

To gain an appreciation of how users perceive the mass, balance and inertia of an object, a study was set-up at the University of Bristol. This was completed using 1st, 3rd and 4th year undergraduate engineering students. The test method is described below.

\subsection{Example part}

For the study, the Ultimaker Drill was used as an example part. This, at the time of writing, can be downloaded from YouMagine under a creative commons licence (Ultimaker, 2017). As part of the study this was remixed to include openings for additional mass to be added. The .stl files for this can be found and downloaded under the same licence at (https://www.youmagine.com/designs/ultimakerhand-drill-with-mass-pockets). All drills were manufactured at $105 \%$ of their standard size.

The drills were printed using Ultimaker 3 Extended printers, with Ultimaker black and white PLA. The print method suggested by Ultimaker was followed, using a $0.1 \mathrm{~mm}$ layer height and $20 \%$ infill, which was changed to a grid design to aid mass addition. Retraction was reduced to avoid grinding of the material.

Three drills were used within the study. These were:

1. A standard drill with no added mass;

2. A drill with mass added to the base of the handle; and,

3. A drill with mass added to the upper section.

For the study, standard $8.5 \mathrm{~mm}$ HSS drill bits were adhered into the "chuck" of the drill. The intention was for the mass properties of the drills to be the only discernible differences between the three artefacts.

\subsection{Mass property manipulation}

The mass properties of Designs 2 and 3 were altered using small lead masses and coarse iron filings. The lead masses were $4.5 \mathrm{~mm}$ diameter pellets with individual masses of 0.45 grams. Their size was dictated by the space available within the infill of the part and what was commercially available. Mass was added to Design 2 post-fabrication by placing the lead and iron in the base of the handle. Mass was added to Design 3 in-process by pausing the build. The lead and iron were placed within the part and the top surface held in place using adhesive. The print was then resumed. The printer was able to continue with no issue after the placement of mass, as shown in Figure 3.

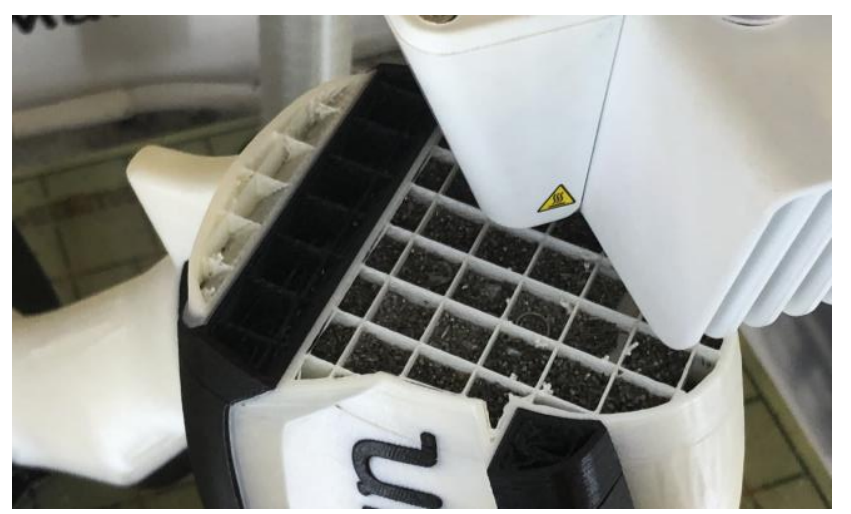

Figure 3. Design 3 being manufactured, showing the addition of mass 
The mass of each drill was not altered to replicate the mass distribution of any design but was done to differentiate the mass properties between each drill. The locations of the Centre of Mass (CoM) for each drill are given in Figure 4. The masses of each design were $0.41 \mathrm{~kg}, 0.47 \mathrm{~kg}$ and $1.01 \mathrm{~kg}$ respectively.

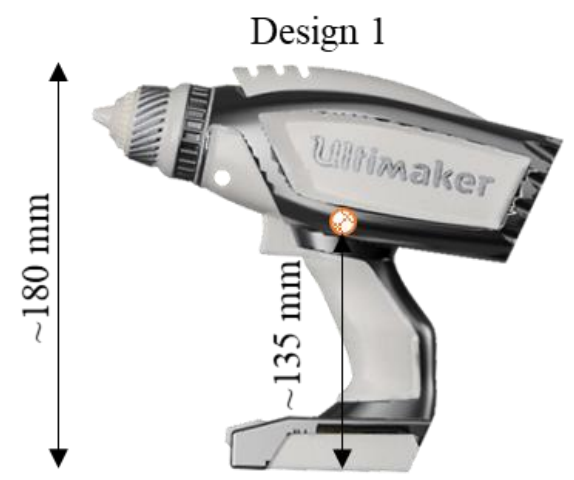

Design 2

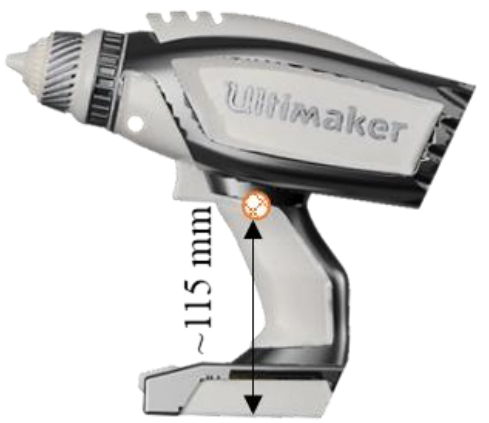

Design 3

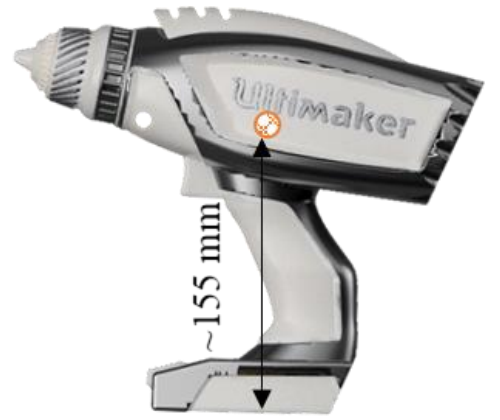

Figure 4. CoM positions with respect to the drill height

\subsection{Task}

With no other participants in the immediate vicinity of the experiment, the participants were asked to complete a short task and then fill in a three-question survey. This task involved using each drill one at a time and placing the drill bit through a $6 \mathrm{~mm}$ acrylic sheet containing six $10 \mathrm{~mm}$ holes. The acrylic sheet was designed such that the drills had to be turned to allow the drill bit to fit through several of the holes. A prize was awarded for the fastest participants with a single and all three drills. This was done to ensure the task was performed with an unambiguous objective by every participant. The task set-up is shown in Figure 5.

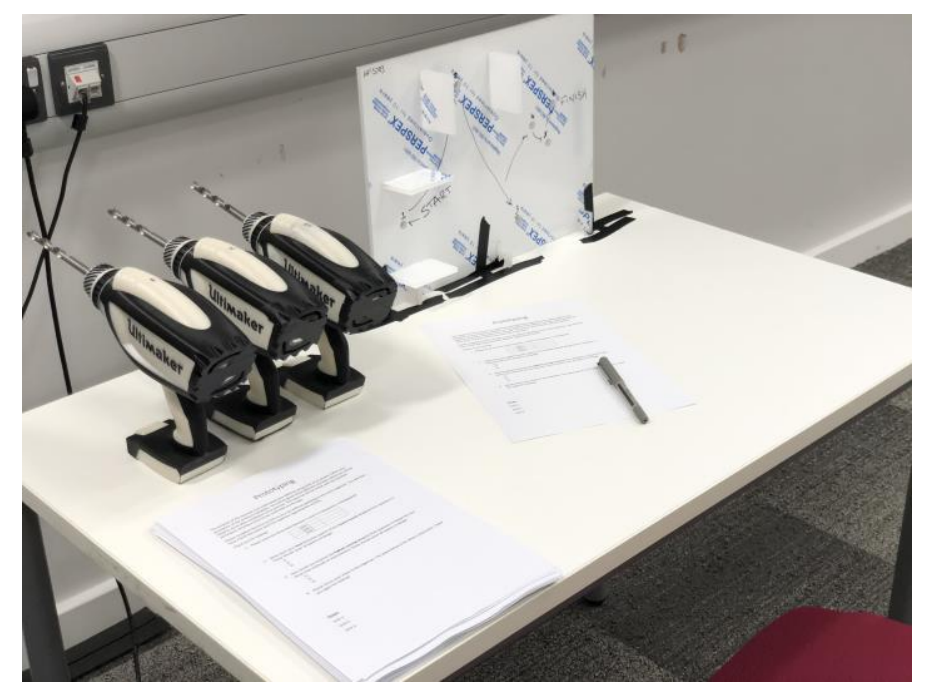

Figure 5. Study set-up

The participants were asked to randomly pick an order to use each drill. This resulted in the distribution shown in Table 2.

Table 2. Drill order summary

\begin{tabular}{|c|c|c|c|}
\hline \multirow{2}{*}{ Design } & \multicolumn{3}{|c|}{ Sequence } \\
\cline { 2 - 4 } & 1 st & 2nd & 3rd \\
\hline 1 & 7 & 8 & 10 \\
\hline 2 & 13 & 8 & 4 \\
\hline 3 & 5 & 9 & 11 \\
\hline
\end{tabular}


The order the drills were used in was well dispersed, except for Design 2 being used first more commonly than any other. The times taken to complete the task with each drill for each participant were recorded. The participants were told the time it took with each drill as the task progressed.

The survey, which was initially filled out independently, included three questions. These were:

1. Please score the three drills between 1 and 5 ( 1 being poor, 5 being good).

2. What were your least favourite aspects of the lowest scored design(s) from question 1? These should cover all aspects of design.

3. How would you improve the highest scoring design(s) from question 1? Please list your three main methods of improvement. These should cover all aspects of design.

After completing the survey, a short exit interview was included in order to resolve any ambiguous answers. The author tried their utmost to not lead any responses, instead asking what they meant by phrases such as "heavy, bulky" and "hard to move precisely", as just two examples.

\subsection{Test sample}

The study had 25 participants, with 18 from 1 st year Mechanical engineering, and seven $3 \mathrm{rd} / 4$ th year students from the same course. The results gathered from each group are indistinguishable from each other, so are presented within this paper together.

\section{Study results}

All the participants completed the survey indicating a single preferred design, though the two lowest scoring designs were rated equal on 3 occasions. However, this was clarified as the participants not liking aspects of either design rather than not being able to tell them apart.

Design 2 was preferred with an average score of 3.6 and was chosen as the best 11 times. This compared to Drills 1 and 3 with scores of 3.16 and 2.88 respectively. As Design 2 was also the drill most commonly selected to be used first, it was checked that the order in which drills were used did not correlate to the order of preference. It was found, through a simple comparison, that the order of use had a negligible effect on the order of preference.

Design 3, which was scored lowest by the participants, was most frequently the fastest drill used to complete the task. It was thought that there would be a perceived benefit by the participants that faster meant better, as there was a prize for being quick. However, even though the contributors were aware of their times throughout the experiment there was little correlation between this and design preference.

From analysis of the results, it was not thought the drill order or task time had perverted the outcomes.

The answers given to questions 2 and 3 were then considered and grouped. These groups related to the three mass-related properties (mass, balance and inertia) and "Other", as well as being considered preand post- discussion. Both sets of results are shown in Figure 6.

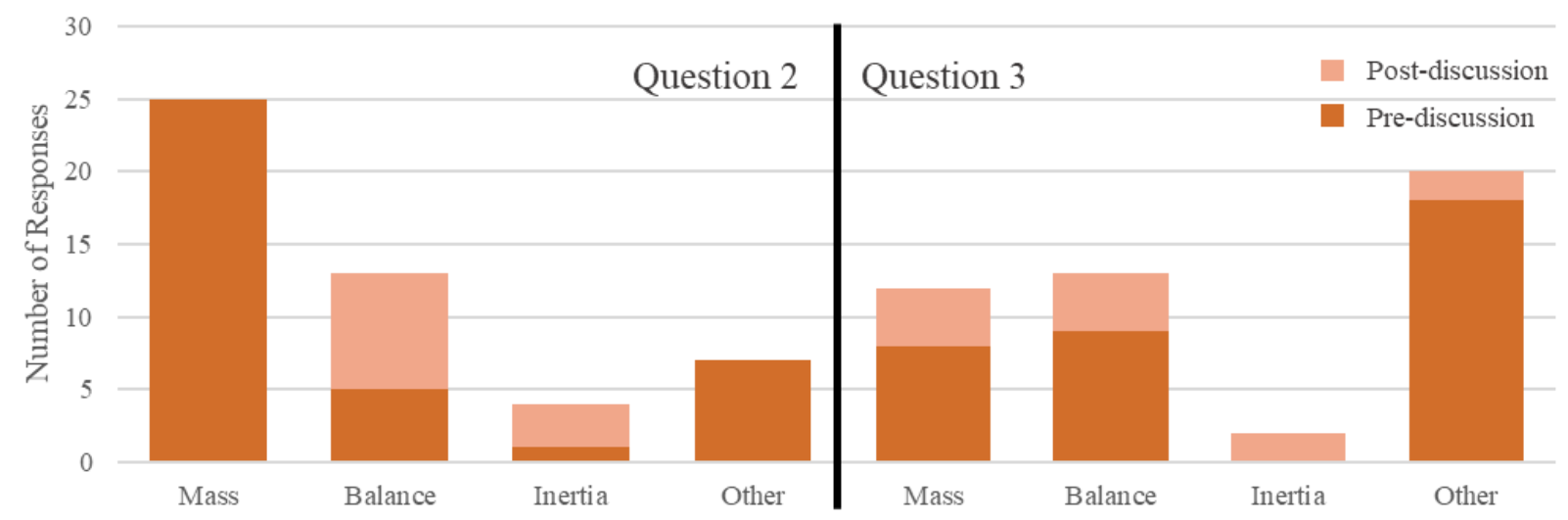

Figure 6. Categorisation of responses to Questions 2 (left) and 3 (right) from the study 
The results from question 2 demonstrated a very clear understanding that mass influenced user perception of the prototypes. All participants mentioned "mass", "heavy", "light" or similar in their answers. A lesser number explicitly mentioned balance, or phrases such as "top heavy", without further clarification; though this came readily once asked. Of the three properties, inertia was highlighted the least by the participants. This was thought to be for two reasons; the effect of inertia was minimal for the given task and it is the most complex property to recognise and describe. That said, inertia was still cited by 4 participants in response to question 2 .

The answers to question 3 posed a similar result to that of questions 2. However, as the question asked for improvements, many participants did not mention mass as they did not feel it needed improving. Balance was commented upon without prompting more readily than in the answers for question 2 . Inertia was again not widely cited/stated, likely due to the same reasons as highlighted previously.

\section{Discussion}

The results from the study demonstrated that mass properties could be recognised by the participants, though balance and inertia were recognised less often than absolute mass. Balance and inertia frequently required some level of discussion before being recognised too. Inertia was commented upon the least of the three mass properties. This is thought to be for several reasons. Firstly, the effect of inertia was thought to be less prevalent than that of mass or balance for the task. Secondly, the consequences of prototypes having different inertias was thought to be less well understood than the effects of the other two properties; this may be due to the participants level of understanding, and future studies should use a more varied pool of participants to understand the effect this has.

Secondly, the design favoured by each participant was interesting. Even with a possible reward available for good performance, participants frequently favoured designs with preferential mass properties than their best performing design. Though this raises several questions into the evaluation criteria that participants were using, it demonstrates that mass properties have a significant effect on user perception.

Third and finally, it is interesting to note that the participants preferred design 2 in $44 \%$ of cases. Design 2 has most the most similar balance as common cordless drills, with the CoM lower due to the larger battery mass. This is likely the drill designs the participants have been most exposed to prior to the experiment, and so a comparison to this may have been drawn. In contradiction, Design 3 has the most similar absolute mass to a mains-powered drill, whilst also having an absolute mass not dissimilar to cordless drills. Despite considering this, and that mass was the most recognised mass property, Design 3 was the least favoured design. As such, it is not thought that comparison to existing designs had a significant effect on the results of the study.

\section{Industry consultation}

To complement and support the study, design companies were consulted to understand how they view the importance of mass properties in prototyping. Two companies were targeted; Amalgam - one of the worldwide leading model makers based in Bristol, UK - and Moulton Bicycle Company manufacturers of premier bicycles in Bradford on Avon. The contributing representatives at these companies were Mike Harvey, Amalgam's director for prototyping, and Dan Farrell, Moulton Bicycle Company's Technical Director.

Three questions were proposed to the two parties, along with space for any additional thoughts. This followed a brief introduction that outlined the aim of the study. The questions were:

1. Would you consider the incorporation of mass distribution into a prototyped artefact a useful feature?

2. Have you and/or your company previously completed any work into replicating the mass distribution of a part (or parts)? If yes, and you can, please provide an example of the part and how you replicated this distribution.

3. Do you have any suggestions on parts or assemblies that may be of interest to you and/or your company, or that may be good examples generally? 
Both companies indicated that the incorporation of mass in a prototype artefact would be useful, with Amalgam stating (in response to question 1):

"Yes. While an early stage prototype might not need to have realistic mass distribution, for many it would be a very useful, if not essential element."

Though early-stage prototypes were highlighted here, this was later expanded to mid-phase prototyping when discussing when best to incorporate realistic mass properties:

"It seems to me that the inclusion of realistic mass etc. is best suited to the mid-stages of a product development cycle. ... late stages would usually include "Real" or bought-in components such as batteries, electronics, motors etc."

It was highlighted that the later stages of prototyping would normally include functional components, which themselves will give the prototype realistic mass properties. Although this does not necessarily impact the development of MEX artefacts, it adds further evidence to the value of mass property replication throughout prototyping.

Moulton Bicycle Company also cited the importance of mass on product prototypes and stakeholder perception within two examples. The first of these highlighted how bikes can feel heavy if the mass distribution is not balanced, as well as highlighting the effect on the bike's handling:

"There is a thing about perception of mass going on, certainly with bicycles. A bike feels heavier if the mass is concentrated at one end rather than balanced. The momentum of an object is also something to consider."

The second example moved away from the "feel" of the prototype and how this affects stakeholder assessment. Instead it was raised that for some products the mass balance is a critical part of the design:

"... a good example is the original Moulton bicycle, where the carrying handle is integral to the frame. For it to work properly the handle has to be at the centre of mass - if you get the mass distribution wrong it doesn't work well or at all."

If a design does not fulfil the original intent or requirement of a product it will be negatively reviewed by stakeholders. Therefore, fully replicating mass properties within a prototype may be a requirement for designs where the mass, balance and/or inertia have a critical role in the success of the design.

Consultation with industry has provided further evidence and validation that mass properties do have a significant effect on product perception and has indicated the phases of prototyping where this would be most useful. It has also highlighted that mass, balance and inertia can be critical in the success of a design and hence consideration during the design process is essential.

\section{Conclusions and future work}

The work detailed within this paper has allowed the following conclusions to be drawn:

1. All participants were able to clearly recognise differences in mass, several could recognise balance variations and a few recognised different inertias;

2. Despite the requirement of completing a task, with reward for performance, many participants were more heavily influenced by mass (rather than task competence), and;

3. Mass is important for user interaction but can also be a design driver - as validated by industry.

Although further studies could expand upon the work, the authors contend that there is enough evidence to research methods of mass property twinning in prototypes for early and mid-phase prototyping. Later stage prototypes should also have relatable mass properties, though these artefacts often benefit from the inclusion of functional components in any case. Specifically, the focus is thought to best be on MEX fabricated prototypes due to their widespread use and current limitations.

To further MEX artefact mass replication, work will need to be completed to understand mass property breakdowns of designs. This applies to both parts and assemblies. Investigation into the technologies capable of automatically incorporating mass into these parts/assemblies should also be carried out. As it is unlikely mass can be placed in the same positions as in the intended design, 
properties may have to be replicated without perfectly reproducing the mass distribution. Example technologies are currently thought to include using automated deposition of dense powders/pellets, graduated infill printing and printing of metal-based filaments within the MEX process. Other technologies may also be considered.

\section{Acknowledgements}

The work reported in this paper has been undertaken as part of the Twinning of digital-physical models during prototyping project at the University of Bristol, which is funded by the Engineering and Physical Sciences Research Council (EPSRC), grant reference (EP/R032696/1).

The authors would also like to thank Mike Harvey and Dan Farrell for their contributions to this work.

\section{References}

Buchenau, M. and Suri, J.F. (2000), "Experience prototyping”, In: Proceedings of the conference on Designing interactive systems processes, practices, methods, and techniques - DIS '00, ACM Press, New York, New York, USA, pp. 424-433. https://doi.org/10.1145/347642.347802

Camburn, B. et al. (2017), "Design prototyping methods: State of the art in strategies, techniques, and guidelines", Design Science, Vol. 3, p. e13. https://doi.org/10.1017/dsj.2017.10

Coutts, E.R., Wodehouse, A. and Robertson, J. (2019), "A Comparison of Contemporary Prototyping Methods", Proceedings of the Design Society: International Conference on Engineering Design, Vol. 1 No. 1, pp. 1313-1322. https://doi.org/10.1017/dsi.2019.137

Desktop Metal (2019), Desktop Metal, Available at: https://www.desktopmetal.com/

Gibson, I., Gao, Z. and Campbell, R.I. (2004), "A comparative study of virtual prototyping and physical prototyping", IJMTM, Vol. 6, pp. 503-522. https://doi.org/10.1504/IJMTM.2004.005931

Houde, S. and Hill, C. (1997), "What do Prototypes Prototype?", In: Handbook of Human-Computer Interaction, Elsevier, pp. 367-381. https://doi.org/10.1016/B978-044481862-1.50082-0

James Dyson Foundation (2019), Dyson Design Process Box, Dyson Design Process Box, Available at: https://www.jamesdysonfoundation.co.uk/content/dam/pdf/FOR WEB DESIGN PROCESS BOX TEACHERS PACK INSIDE_Single Pages_Updated New.pdf? (Accessed: 4 October 2019).

Jensen, L.S., Özkil, A.G. and Mortensen, N.H. (2016), "Prototypes in engineering design: Definitions and strategies", Proceedings of International Design Conference, DESIGN, Vol. DS 84, pp. 821-830.

Lennings, L. (1997), Selecting Either Layered Manufacturing or CNC Machining to Build Your Prototype, pp. 1-9. Available at: www.spline.nl

Markforged (2019), Markforged Metal X. Available at: https://markforged.com/metal-x/

Mathias, D. et al. (2018), "Characterising the affordances and limitations of common prototyping techniques to support the early stages of product development", Proceedings of International Design Conference, DESIGN, Vol. 3, pp. 1257-1268. https://doi.org/10.21278/idc.2018.0445

Mathias, D. (2018), Hybrid Prototyping - Physical Implementation, Design and Manufacturing Futures Lab.

Mathias, D. et al. (2019), "Accelerating product prototyping through hybrid methods: Coupling 3D printing and LEGO”, Design Studies. https://doi.org/10.1016/j.destud.2019.04.003

Moreau, C. (2018), "Sculpteo the state of 3D printing 2018", Sculpteo the state of 3D printing 2018, pp. 1-40.

Schneider, K. (1995), "Prototypes as assets, not toys why and how to extract knowledge from prototypes", Proceedings - International Conference on Software Engineering, pp. 522-531.

Simonot, A., Cassaignau, A. and Coré-Baillais, M. (2019), The State of 3D Printing - Sculpteo, Available at: http://www.sculpteo.com/static/0.30.0-62/download/report/Sculpteo_State_of_3D_Printing.pdf

Ultimaker (2017), Ultimaker Drill, Available at: https://www.youmagine.com/designs/hand-drill (Accessed: 23 September 2019).

Ultimaker (2019), Ultimaker - Product Development, Product Development, Available at: https://ultimaker.com/ solutions/product-development (Accessed: 4 October 2019).

Viswanathan, V.K. and Linsey, J.S. (2013), "Role of Sunk cost in engineering idea generation: An experimental investigation", Journal of Mechanical Design, Transactions of the ASME, Vol. 135 No. 12, pp. 1-12. https://doi.org/10.1115/1.4025290

Yang, M.C. (2004), "An Examination of Prototyping and Design Outocome", Proceedings of DETC'04 ASME 2004 Design Engineering Technical Conferences and Proceedings of DETC 20042004 ASME Design Engineering Technical Conferences, pp. 1-6. 